\title{
HIGH RESOLUTION EXAMINATION OF BIOLOGICAL SAMPLES USING FIELD EMISSION SCANNING ELECTRON MICROSCOPY
}

\author{
S. Erlandsen*, J. Detry**, C. Ottenwaelter*, and C. Frethem* \\ *Department of Genetics, Cell Biology, and Development, University of Minnesota Medical School, \\ Minneapolis, MN, 55455 \\ **Honeywell, Plymouth, MN 55447
}

The development of field emission SEM (FESEM) in the last two decades has revolutionized the examination of biological samples. Advances in the design of field emission guns (cold field and Schottkey guns), the improvements attained with immersion lens design for both in-lens and below-the-lens instruments, and the preparation of thin metal coatings $(\sim 1 \mathrm{~nm})$ all contributed to rapid advances in achieving high resolution imaging of features on biological surfaces.

Resolving power of a SEM is related to the size of the electron probe rastered over the sample, and probe size is usually inversely proportional to $\mathrm{keV}$. High voltage provides smaller probe size (improves resolution) but also produces more SE2 which reduce contrast. Low voltage FESEM, defined as less than $5 \mathrm{keV}$, has been proposed (1) as desirable for examination of biological samples due to decreased penetration of the sample surface by the primary beam, resulting in higher contrast of surface features due to a reduction in SE2. Use of low voltage $(<5$ $\mathrm{keV}$ ) FESEM seems to result in less specimen contamination and radiation damage when compared to use of higher keV (1).

Biological samples for FESEM can be divided into two main types: a) infinitely thin, and b) bulk samples. Infinitely thin samples, e.g. viruses, macromolecules, can be examined at higher $\mathrm{keV}$ due to the thinness of the samples $(<50 \mathrm{~nm})$ and are usually coated with chromium for SE imaging. Bulk samples, e.g. cells, tissues, can be examined by SE imaging but often show signs of charging artifact. Advances in BSE detectors permit examination of bulk biological samples with simultaneous imaging of coating metals together with discrimination of high atomic number tracers, such as colloidal gold (Figs. 1-4).

Development of the double coating method of Walther et al $(2,3)$ together with cryo-immobilization of specimens have facilitated examination of molecular topography (Fig. 3). Retention of the shell of hydration on surface molecules prevents molecular collapse seen with complete freeze-drying. The double coating method permits use of higher $\mathrm{keV}$ for improved BSE imaging with smaller probe diameters, and problems frequently encountered in specimen damage in cryo-SEM, e.g. signs of cracking and contamination, are seen to a much lesser degree with this double coating method.

BSE imaging can be accomplished by different types of BSE detectors: 1) solid state silicon or proprietary phosphorus detectors are the least costly, 2) YAG crystals doped with cerium are expensive, but offer the highest resolution, or 3) newly introduced ExB filters function well, particularly at low magnification, at very low voltages $(\sim 1 \mathrm{keV})$ where silicon and YAG are insensitive due to threshold limitations (Figs. 5-6). Due to low BSE yield, chromium is not a desirable coating for high resolution BSE imaging and Pt or W are preferred. Detection of colloidal gold ( $\sim 10 \mathrm{~nm}$ diameter) can be accomplished routinely in FESEM at magnifications $<50,000 \mathrm{X}$ with most BSE detectors using 3-5 keV. High resolution BSE imaging at high magnification $(>100,000 \mathrm{X})$ is optimal with the YAG detector (Fig. 4).

Detection of colloidal gold probes with BSE has become routine in low voltage FESEM, and in conjunction with cryo-immobilization methods, FESEM can now provide direct information about molecular topography of cell surface molecules.

(1) J. Pawley et al The Science of Biological Specimen Preparation for Microscopy and Microanalysis, Scan. Micros. Intl., Chicago, 1989.

(2) P. Walther et al, Scanning 19 (1997) 343.

(3) S. Erlandsen, BioTechniques 31 (2001) 300. 


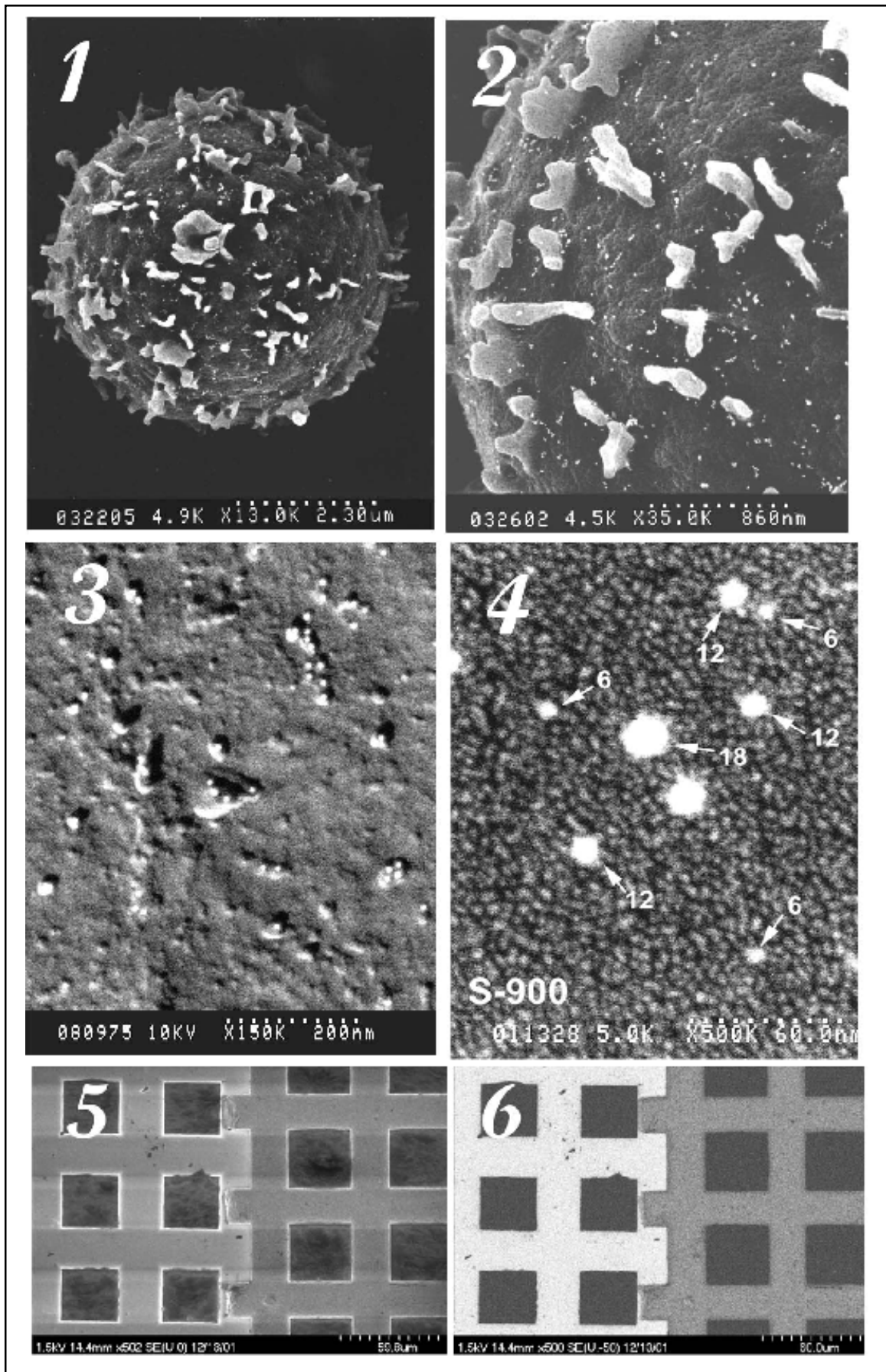

Figures 1 and 2. Low voltage FESEM immunogold localization (10nm) of CD43 on human neutrophil by backscatter electron imaging (YAG detector). Figure 3. Double layer coating of cryo-immobilized human platelet showing immunogold localization (10nm) of P-selectin molecules on membrane surface (YAG detector). Figure 4. Colloidal gold standard coated with $\sim 1 \mathrm{~nm}$ of Pt and imaged by BSE with YAG detector in an in-lens FESEM. Figures 5 and 6. Test grid of gold and copper imaged at $1.5 \mathrm{keV}$ by SE in figure 5, or in figure 6 with ExB filter $(-50 \mathrm{eV})$ in a S-4700 FESEM. The gold grid is on the left in each figure. 